\title{
Remoção do cisto sinovial da articulação atlantoaxial pela via póstero-lateral transdural
}

\author{
André de Macedo Bianco*, Lucas Madeira*, Ida Fortini*, Alexandre José Reis Elias ${ }^{\star * *}$, \\ Milton K. Shibata*** \\ Serviço de Neurocirurgia do Hospital 9 de Julho de São Paulo
}

\section{RESUMO}

Mielopatia cervical alta causada por cisto sinovial da articulação atlantoaxial é uma entidade rara e muito pouco descrita na literatura. Os autores descreveram um caso de cisto sinovial localizado posteriormente ao processo odontóide causando compressão bulbo-medular em paciente com 88 anos de idade. O cisto foi removido através de um acesso póstero-lateral direito com hemilaminectomia de $\mathrm{C} 1$ e ressecção microcirúrgica transdural. A paciente apresentou uma excelente recuperação. Os aspectos etiológicos e terapêuticos são discutidos.

\section{PALAVRAS-CHAVE}

Cisto sinovial. Articulação atlantoaxial. Mielopatia cervical.

\section{ABSTRACT \\ Synovial cyst of the atlantoaxial joint are discussed. \\ KEYWORDS \\ Synovial cyst. Atlantoaxial joint. Cervical myelopathy.}

Upper cervical myelopathy due to synovial cyst of the atlantoaxial joint is a rare condition. The authors report a case of synovial cyst located in the posterior portion of the odontoid process causing cervicomedular compression in a 88 year-old female patient. The synovial cyst was removed via right posterolateral approach, C1 hemilaminectomy and transdural microsurgical resection. The patient presented an excellent recovery. The etiological and therapeutic aspects

\section{Introdução}

Cisto sinovial é um achado comum na pratica médica e, teoricamente, pode ocorrer em qualquer articulação. Freqüentemente, é encontrado na coluna vertebral, principalmente na região lombar, onde se origina na faceta articular. $\mathrm{O}$ acometimento da região raquiana torácica e cervical é menos comum, porém, quando ocorre, pode ser causa de compressão medular. Mielopatia cervical alta resultante de compressão medular por cisto sinovial localizado na articulação atlantoaxial é de ocorrência rara e pouco descrita na literatura. O diagnóstico diferencial inclui lesões como tumores, pannus, subluxação crônica e ectasia da artéria vertebral ${ }^{4,8,16}$.

É relatado um caso de cisto sinovial localizado posteriormente ao processo odontóide do axis, causando compressão medular anterior. Após descom- pressão e retirada da lesão, a paciente apresentou uma excelente recuperação.

\section{Relato do caso}

HS, 88 anos, sexo feminino. Hipertensa e diabética. Queixava-se de dor na região posterior do pescoço e parte póstero-lateral direita da cabeça há quatro anos, que se iniciou após acidente automobilístico. Seis meses antes da internação, sofreu novo acidente automobilístico após o qual passou a sentir dormência e formigamento no membro superior direito e nos membros inferiores e dificuldade à deambulação, necessitando de apoio para manter-se em pé.

\footnotetext{
*Residente de Neurocirurgia.

** Neurologista.

*** Neurocirurgião.
} 
Ao exame apresentava um discreto déficit motor (força grau IV) no membro superior direito (MSD), paraparesia crural e hiporreflexia bicipital e estilorradial à direita associado a uma hemi-hipoestesia completa à esquerda. A ressonância magnética (RM) da coluna cervical evidenciou lesão intrarraquiana extramedular localizada posteriormente ao odontóide e anteriormente à transição bulbo-medular, determinando compressão importante neste nível. Apresentava hipossinal nas imagens ponderadas em T1 e hipersinal em T2; após administração de contraste, houve um discreto realce periférico (Figuras 1, 2 e 3).

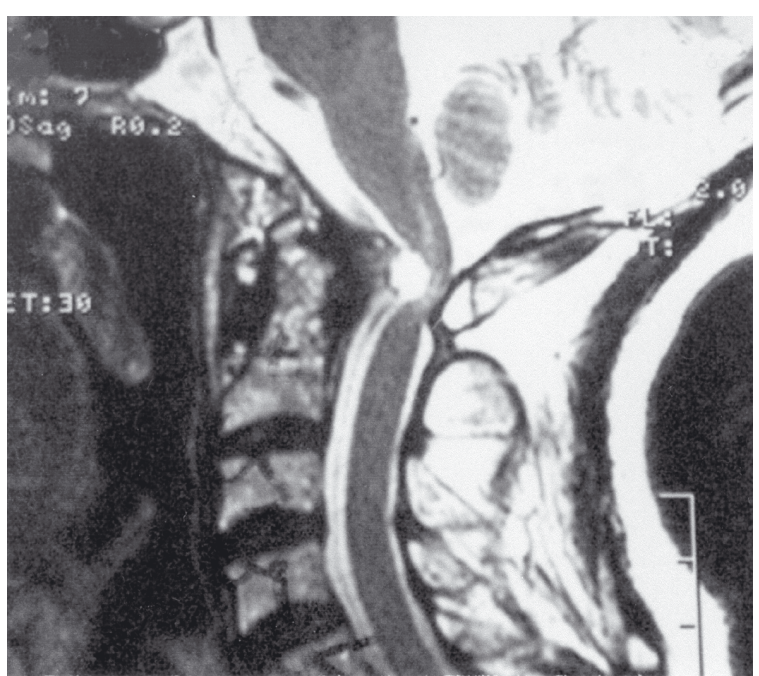

Figura 1 - Ressonância magnética em T2 sagital demonstrando lesão com hipersinal localizada posteriormente ao processo odontóide do axis exercendo compressão importante ao nível da transição bulbo-medular.

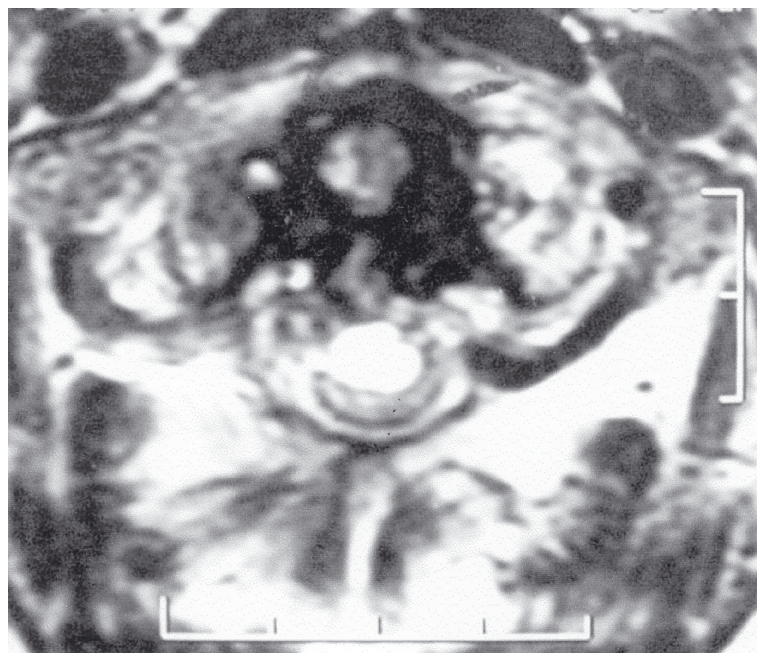

Figura 2 - Ressonância magnética em T2, mostrando, em corte axial, a lesão comprimindo a medula.

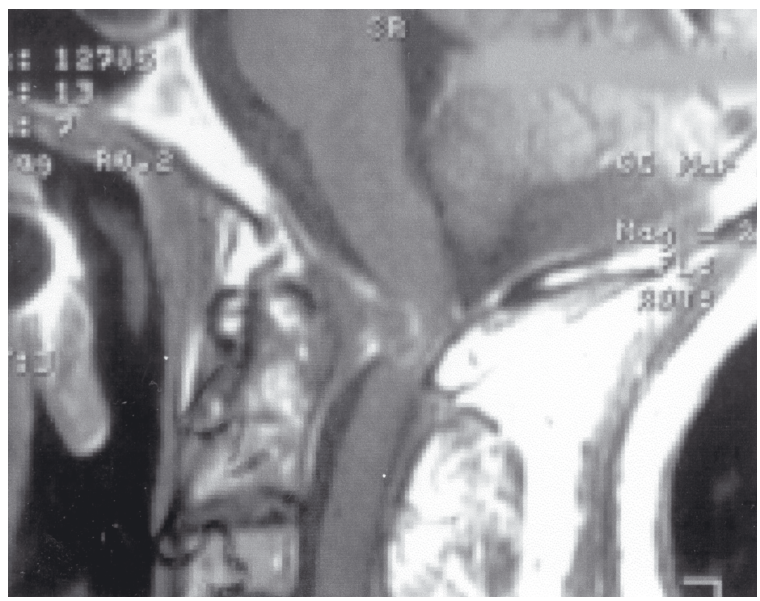

Figura 3 - Ressonância magnética, após administração de gadolíneo demonstrando a lesão hipointensa em T1, com discreto realce periférico.

A exérese da lesão foi realizada através de acesso cervical póstero-lateral com a paciente em decúbito lateral esquerdo. $\mathrm{O}$ arco de $\mathrm{C} 1$ foi retirado e uma incisão longitudinal e paramediana, com cerca de $2 \mathrm{~cm}$, foi feita na dura-máter. A lesão pôde ser alcançada sem retração da medula, seccionado-se os ligamentos denteados expostos. Após ressecção microcirúrgica transdural do cisto, a medula tornou-se livre de compressão.

No pós-operatório imediato evoluiu com uma piora da hemiparesia direita que melhorou parcialmente em sete dias, quando recebeu alta hospitalar. O exame anatomopatológico foi compatível com cisto sinovial. $\mathrm{Na} \mathrm{RM}$ realizada seis meses após a operação, observa-se que a ressecção da lesão foi total e que não há mais compressão medular (Figura 4). Um ano após o tratamento cirúrgico, a paciente encontra-se sem déficits e assintomática.

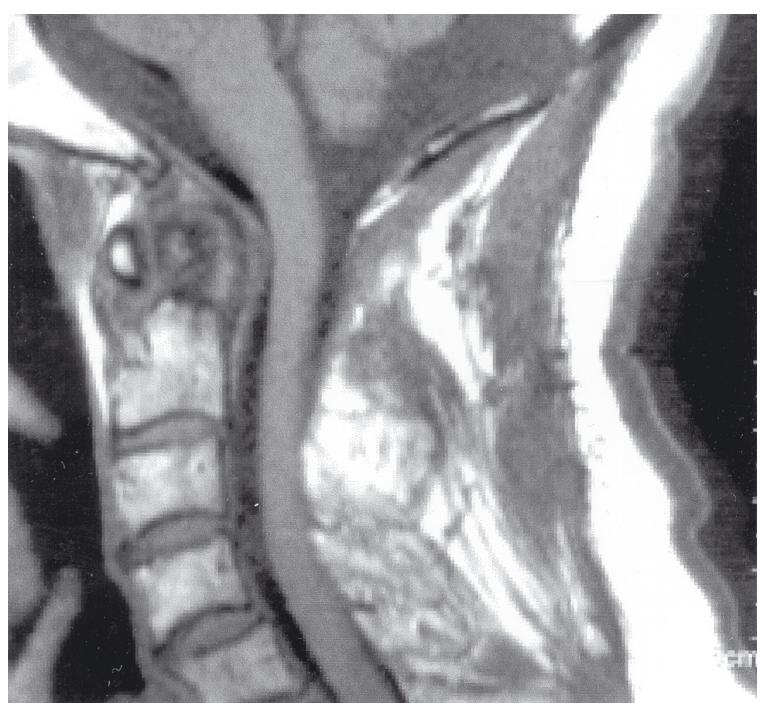

Figura 4 - Ressonância magnética em T1 seis meses após o tratamento. Nota-se remoção total do cisto e descompressão medular adequada. 


\section{Discussão}

Os cistos sinoviais intra-espinais têm sido cada vez mais diagnosticados e são causa comum de radiculopatias. Ocorrem principalmente em áreas de grande mobilidade e, geralmente, são adjacentes às facetas articulares, localizando-se lateral ou dorsolateralmente no canal espinal. A região lombar é a mais acometida ${ }^{6,10}$.

O acometimento da região cervical foi inicialmente descrito, em 1974, por Kao e cols. ${ }^{9}$ Em 1988, Onofrio e Mih $^{13}$ descreveram uma forma especial de cisto sinovial envolvendo o ligamento cruciforme do odontóide. A partir daí, pouquíssimos relatos similares têm sido publicados ${ }^{1-5,11,16}$.

Goffin e cols. ${ }^{5}$ propuseram o termo "cisto articular degenerativo espinal", pois essas lesões apresentam relação anatômica com alguma estrutura articular, e a patogênese está relacionada com alterações degenerativas da articulação.

Várias teorias têm sido propostas para explicar a existência de cisto sinovial espinal, incluindo metaplasia, presença de restos embrionários, degeneração póstraumática, estresse da faceta articular com herniação de tecido sinovial, degeneração mucinosa do tecido conjuntivo, aumento da produção de ácido hialurônico por fibroblastos e proliferação inespecífica de células mesenquimais ${ }^{1}$. Suportado pela observação de que a maior parte dos cistos sinoviais desenvolvem-se ao nível de L4-L5, o qual apresenta maior mobilidade, muitos autores acreditam que o excesso de estresse ao nível da faceta articular é um importante fator na formação cística ${ }^{1,7,12,14}$.

Fransen e cols. ${ }^{4}$ relataram um caso de degeneração cística do ligamento transverso do processo odontóide causando compressão da transição bulbo-medular. Esses autores, após revisão da literatura, caracterizaram a sintomatologia de síndrome degenerativa de compressão anterior da medula espinal que consistia em distúrbio da marcha, parestesias de membros superiores e dor nos braços e no pescoço, geralmente ocorrendo em pacientes com mais de 60 anos e que apresentavam osteoartrite. Posteriormente, Zorzon e cols. ${ }^{16}$ caracterizaram a síndrome do cisto sinovial ou do cisto articular degenerativo espinal da articulação atlantoaxial como ocorrendo geralmente em pacientes com mais de 60 anos, sem história pregressa de trauma, sem evidência de artrite reumatóide, com sinais e sintomas de compressão da medula cervical de início subagudo. A RM pode, às vezes, demonstrar uma captação periférica do contraste, e o exame histológico evidencia pouca atividade inflamatória. $\mathrm{O}$ tratamento é cirúrgico e apresenta bons resultados e nenhum relato de recorrência.

Dois casos de cisto sinovial associado à instabilidade da junção craniocervical foram descritos ${ }^{1,3}$. Em um, a lesão foi retirada pela via transcondilar associada à craniotomia suboccipital lateral, seguido de fusão com enxerto ilíaco e instrumentação ${ }^{1}$. No outro, a lesão regrediu espontaneamente após laminectomia de $\mathrm{C} 1$ C2 seguido de fusão - e este foi o único caso com antecedente traumático ${ }^{3}$. Os autores, deste último caso, atribuíram o desenvolvimento do cisto como resultado da degeneração do ligamento transverso em virtude da instabilidade da articulação atlantoaxial.

A maioria dos casos descritos não apresenta relação entre a lesão e um antecedente traumático. $\mathrm{O}$ caso relatado por nós apresenta uma íntima associação com traumatismo. A paciente sofreu dois acidentes automobilísticos. Um ocorreu quatro anos antes do tratamento cirúrgico, no qual a paciente apresentou somente sintomatologia álgica local. E no segundo acidente, seis meses antes de procurar auxílio médico, a paciente apresentou parestesia no membro superior direito e nos membros inferiores. Durante investigação, evidenciouse compressão medular provocada por material sinovial, provavelmente em decorrência da herniação do tecido sinovial localizado entre a porção transversa do ligamento cruzado e o odontóide. Acreditamos que o antecedente traumático tenha causado alguma lesão na articulação atlantoaxial propiciando o aparecimento do cisto. Como o quadro clínico apresentou características evolutivas, existe um forte indício de aumento progressivo do cisto. É importante ressaltar que o trauma sofrido não foi de grande magnitude. Nos poucos casos descritos na literatura, a etiologia traumática tem sido descartada. No entanto, é possível que trauma leve pregresso não seja lembrado pelo paciente e, algumas vezes, passar despercebido na história médica.

As vias de acesso utilizadas nos casos descritos na literatura são a transoral seguida de fixação posterior e póstero-lateral com hemilaminectomia de $\mathrm{C} 1$ ou $\mathrm{C} 1$ $\mathrm{C} 2$ com ou sem descompressão do forame magno ${ }^{15,16}$. Em um relato, os autores realizaram fusão craniocervical posterior após hemilaminectomia de $\mathrm{C} 1$ por ter sido constatada instabilidade ${ }^{1}$.

Em virtude da idade da paciente, optamos por acesso cirúrgico menos agressivo, pela via pósterolateral com hemilaminectomia de $\mathrm{C} 1$, que possibilitou com sucesso a ressecção microcirúrgica transdural do cisto. Não houve necessidade de fixações e artrodeses. É uma via mais conservadora com menor número de complicações pós-operatórias.

\section{Referências}

1. AKIYAMA H, TAMAKI N, KONDOH T, NAGASHIMA T: Craniocervical junction sybovial cyst associated with atlanto-axial dislocation - case report. Neurol Med Chir 39:539-43, 1999. 
2. BIRCH BD, KHANDJI AG, MCCORMICIK PC: Atlantoaxial degenerative articular cyst. J Neurosurg 85:810-6, 1996.

3. CHANG H, PARK JB, KIM KW: Synovial Cyst of the transverse ligament of the atlas in a patient with os odontoideum and atlantoaxial instability. Spine 25:741-6, 2000

4. FRANSEN P, PIZZOLATO GP, OTTEN P, REVERDIN A, LAGIER R, TRIBOLET N: Synovial cyst and degeneration of the transverse ligament: na unusual cause of hight cervical myelopathy. J Neurosurg 86:1027-30, 1997.

5. GOFFIN J, WILMS G, PLETS C, BRUNEEL B, CASSELMAN J: Synovial Cyst at the C1-C2 junction. Neurosurgery 30:914-6, 1992

6. HOWINGTON JU, CONNOLLY ES, VOORHIES RM: Intraspinal synovial cysts: 10-year experience at the Ochsner Clinic. J Neurosurg 91:193-9, 1999.

7. JABRE A, SHAHBABIAN S, KELLER JT: Synovial cyst of the cervical spine. Neurosurgery 20:316-8, 1987.

8. KAINBERGER F, TRATTNIG S, CZERNY C, SEIDEL G, KRITZ H, IMHOF H: MRI in Assessment of the Systemic Manifestation of Rheumatological Disease. $\mathrm{Br}$ J Rheumatol 35:40-4, 1996.

9. KAO CC, WINKLER SS, TURNER JH: Synovial cyst of the spinal facet. Case report. J Neurosurg 41:372-6, 1974.

10. LYONS MK, ATKINSON JL, WHAREN RE, DEEN HG, ZIMMERMAN RS, LEMENS SM: Surgical evaluation and manegement of lumbar synovial cysts: the Mayo Clinic experienxe. J Neurosurg 93:53-7, 2000.
11. MilleR JD, AL-MEFTy O, MIDDLETON III TH: Synovial cyst at the craniovertebral junction. Surg Neurol 31:239-42, 1989.

12. NIJENSOHN E, RUSSEL EJL, MILAN M, BROWN T: Calcified synovial cyst of the cervical spine: CT and MRI evaluation. J Comput Assist Tomogr 14:473-6, 1990.

13. ONOFRIO BM, MIH AD: Synovial cysts of the spine. Neurosurgery 22:642-7, 1988.

14. PATEL SC, SANDERS WP: Synovial cyst of the cervical spine: case report and review of the literature. AJNR 9:602-3, 1988

15. SALAS E, SEKHAR LN, ZIYAL IM, CAPUTY AJ, WRIGHT DC: Variations of the extreme-lateral craniocervical approach: anatomical study and clinical analyisis of 69 patients. J Neurosurg (Spine 2) 90:206-19, 1999.

16. ZORZON M, SKRAP M, DIODATO S, NASUELLI D, LUCCI B: Cysts of the atlantoaxial joint: excellent long-term outcome after posterolateral surgical decompression. J Neurosurg 95:111-4, 2001.

Original recebido em agosto de 2002

Aceito para publicação em dezembro de 2002

\section{Endereço para correspondência:}

André de Macedo Bianco

Rua Peixoto Gomide, 613 - $9^{\circ}$ andar

CEP 01409-001 - São Paulo, SP

E-mail:ambianco@terra.com.br 